\title{
Methods for Limiting the Calculation Area During Problem Solving by the Finite Difference Method
}

\author{
S. A. Marković, J. M. Cvetić, T. D. Koledin
}

\begin{abstract}
By using the integro-differential approach and classical boundary conditions (such as Dirichlet's, Neumann's or the very rarely used Cauchy boundary condition) for solving the two-dimensional problems in open space by the finite difference method, it is possible to - in the numerically exact way - close the calculation area to finite distance. Thus, one of great limitations of the finite difference method is overcome.
\end{abstract}

Keywords: boundary conditions, finite difference method, open space, numerically exact

\section{Introduction}

Various problems of electromagnetics are solved by number of numerical methods, among which is the finite difference method, particularly suitable for solving the partial differential equations. Out of the set group of problems, particular attention will be paid to those in free space. Those problems are characterized by the fact that the area of calculation is necessarily limited by enclosing surface (boundary surface) in space, and the whole area is covered with a grid suitable for the implementation of selected numerical methods. Under the condition that the boundary surface is sufficiently distant from the field source, the corresponding boundary conditions are given in order to obtain a unique solution (for the sake of orientation, the atribute "sufficient" commonly means that the boundary surface is distanced at least two to three maximal diameters of the surface which encloses all the field sources). For instance, in dynamical problems the radiation bounding conditions (Sommerfeld or radiation conditions of higher order) are commonly given; in magnetostatics - it is the Dirichlet's condition, or less commonly, Neumann's homogenous condition; in electrostatics - it is the Dirichlet's condition in the form of $V=$ const. and/or Neumann's condition etc.

The above mentioned conditions are - in general - approximative, because the boundary surface would naturally be in the infinity and not at the limited distance from the field

\footnotetext{
Manuscript received July 15, 2013 ; accepted October 21, 2013

S. A. Marković, J. M. Cvetić and T. D. Koledin are with the Faculy of Electronical Engineering, Belgrade, Serbia
} 
source. However, by moving the boundary surface towards the infinity, the boundary conditions become correct and in that case the problem is not numerically solvable because the grid covering the area becomes infinite. Conflicting demands are linked both to the boundary position and to the exact boundary conditions, from one side, and the numerical solvability of the problem, from the other side, show the still for seeking the solution. The boundary surface obviously must not be in the infinity, and the boundary conditions must - if possible - be exactly determined on the boundary moved to the finite distance from the field source.

The process of redefining the boundary conditions is possible to implement. Both this process and solving the particular problem will be demonstrated on two-dimensional electrostatic example in several equivalent variants. The condition that makes the distance between the boundary surface and the field source sufficient does not need to be so strict anymore, which enables the grid-covered area to be much smaller than the grid usually used in common procedures with approximate boundary conditions. This demonstrates that using the numerical solving with new boundary conditions is faster and more convenient.

\section{Overview of existing methods for limiting the calculation area in two-dimensional problems}

One of the most used ways for limiting the area is the introduction of an artificial boundary at the sufficient large distance from the field source and the assignment of either one of the two conditions (the Dirichlet's or Neumann's) on the boundary, or a combination thereof boundary, where the conditions are in the form

$$
V=V_{0} \text { or } \frac{\partial V}{\partial n}=k,
$$

where $n$ is a normal to the boundary surface and $k=$ const.

The other way starts from the physical nature of the potential function in two-dimensional problems. The asymptotic expression for the potential function of a point at a large distance $r$ from the field source is

$$
V=A \ln \frac{r}{r_{0}}
$$

where $A=$ const., and $r_{0}$ is the distance from the reference point in which is $r_{0} \ll r$. From eq. (2), the relation between the potential function and its derivative by $r$ follows

$$
\frac{\partial V}{\partial r}=\frac{V}{r \ln \left(\frac{r}{r_{0}}\right)}
$$

which is recognized as the Roben's homogenous condition [1].

Both mentioned methods for limiting the grid in order to further implement the finite difference method (or the finite elements method) have one serious lack of consistency mentioned in the introduction, namely the artificial transfer of the boundary from the infinity to 
the finite distance. It is obvious that limiting the domain means that the original problems is substituted by a somehow similar problem, and that the final solution will contain two types of errors, one that originates from the problem substitution and the other which each numerical method incorporates into the solution. The former error is impossible to estimate beforehand. As for the other error, it could be estimated relatively easy either by the implementation of the finite difference method of higher order or by shrinking the dimensions of cells in the grid.

The final estimate of the quality of the solution is a very delicate question because it is difficult to evaluate which one of the two errors will become dominant.

There is another way for the boundary surface, i.e. the curve, to be moved (transferred) from infinity to a finite distance and the grid to be bounded. It is the combined implementation of the two numerical methods, the finite difference method and the method of fictitious load method. Inside the calculation area and in the vicinity of the boundary surface fictitious charges of unknown linear density are suitably placed; the densities being determined from both the potential continuity condition and the condition of normal components of the electric field vector on boundary line which encloses the mesh of the elements [5, 6]. For the calculation of the field and other variables inside the grid, the solution obtained by the finite elements method is used; for the calculation outside of the mesh the fictitious loads are used.

The last way of limiting the grid of elements represents the combination of two numerical methods - requires the domain to be limited and the other one has no similar limitations. It is not difficult to conclude that the role could also be filled by the method of integral equations which too, does not need a finite domain. In the same way, the role of the finite elements method might be filled by the finite differences method. These two methods completely overlap in some special cases [11].

Unlike the method of artificial domain limitation and direct implementation of boundary conditions of the first, second or third kinds on the new boundary, the combined methods of finite differences (or elements) and methods of fictitious charges (or integral equations) are theoretically correct. The only remaining error is the one inherent in numerical calculations. At least theoretically, it can be reduced at will but it is unavoidable.

\section{Solving of electrostatics problems by using the finite difference method}

A great number of electrostatic problems is commonly reduced to the solving of Laplace/ Poisson elliptical differential equations with corresponding boundary conditions defined for each problem. In two-dimensional problems, the boundary conditions are practically given on the curve which completely encloses the calculation area. The boundary conditions of the first, second and the third kinds, which are given in the points on the boundary curve, are known in the literature as the Dirichlet's, Neumann's and Robin's (or Churchill's [3]) conditions, respectively. Depending on the physical conditions, the parts of the boundary curve or even the whole curve itself can also be in the infinity. In reality, fulfilling the boundary conditions in the points on the boundary curve means that the influence of the 
whole space outside the calculation area is simulated.

In order to numerically solve the electrostatic problem by using the finite difference method, the domain should be limited. Each point on the boundary curve is limited distance from the field source. In other words, the grid covering the calculation area in the finite difference method has finite number of nodes in which the potentials are calculated numerically, e.g. by solving the Laplace's equation

$$
\frac{\partial^{2} V}{\partial x^{2}}+\frac{\partial^{2} V}{\partial y^{2}}=0
$$

When the finite difference method of the first kind is implemented on the mesh with a square cells of dimension $\Delta \times \Delta$, solving of the eq. (4) is reduced to the calculation of the potential in each node $(i, j)$ of the mesh by the equation

$$
V_{i, j}=\frac{\left(V_{i-1, j}+V_{i+1, j}+V_{i, j-1}+V_{i, j+1}\right)}{4} .
$$

For the nodes on the boundary, the eq. (5) does not hold. Limiting the grid is understandable because the finite grid possesses limited number of unknown potentials (in the nodes) which could be calculated by the solving of corresponding system of linear equations, iteratively or by other direct method.

So, for numerical solving of electrostatics problems where the boundary curve is partially or completely in the infinity, it is of special importance to know how to reduce the calculation area from infinite to finite. In that way, one should take care that the shrinking of the grid influences the calculation accuracy as little as possible, or not at all.

\section{A new approach for numerically exact limiting of the calculation area}

For easier understanding of the method, it is assumed that the charge distribution in the space, bounding by the curve in the plane and divided by a grid suitable for the implementation of the finite difference method, is known. After calculating the charge distribution by implementing the integral equations method, the potentials can be solved for all points in space as well as in the nodes of the boundary curve.

The potential distribution in the nodes of the grid would be calculated using the following order. Before the beginning of the iterative process, starting values of the potential are given to all nodes. After one iteration, knowing the potential values, the surface densities of the charges on all surfaces in the domain of the calculation can be estimated. Based on the known distribution of charges obtained by the integral equations method, new values of the potential are calculated in the boundary curve nodes and the iterative procedure by eq. (5) is continued in the same way. First, the new values of the potential in the grid nodes are calculated, then the surface density of charges are calculated - on surfaces of interest, and in the end the potential values in the nodes on the boundary are corrected by the integral equations method. If the iterative procedure converges, the numerically exact solution is obtained. 
The described method is the combination of both differential and integral methods and tacitly implemented boundary condition is of Dirichlet's type. Besides the Dirichlet's boundary condition, the other boundary conditions can be implemented, like Neumann's, Robin's and Cauchy's, as well as the boundary conditions that derive from the equivalence theorem [7]. It should be expected that the numerical results, obtained by the implementation of different boundary conditions, will somehow differ in spite of being numerically exact.

\subsection{Limiting the mesh by implementation of the Dirichlet's boundary condition}

The implementation of the Dirichlet's boundary condition will be shown on the example of a symmetrical two-wire power line of a square cross-section in the air, as shown in Fig. 1. The dimensions of the conductor profiles, the dimensions of boundary surfaces and the conductor potentials are shown in Figs. 1 and 2. The grid for the implementation of the finite difference method is with cell dimensions, $\Delta \times \Delta$ (Fig. 2). The starting values of the potential in all nodes of the grid are $0.5 \mathrm{~V}$, except in the nodes which are on the surfaces of the conductors. The potentials of the conductors are $1 \mathrm{~V}$ and $-1 \mathrm{~V}$.

After the first iteration, the surface density of the charges on the conductor surface was calculated (the density is constant between two nodes on the conductor surface!). The calculation of the surface density is shown in Fig. 3, which represents the detail of Fig. 2 , where only six nodes of the grid are shown on the conductor surface. In the only two cell centers shown in Fig. 3, the intensity of the electrical field component normal to the conductor surface is calculated, and the electric field value on the conductor surface is determined by extrapolation. The surface density of the charges $\sigma$ is calculated as $\sigma=$ $\varepsilon_{0} E$, this is shown in Fig. 3. The surface density of the charges is constant and different between every two nodes on the conductor surface, which means that the conductor surface is approximated with $n$ equally charged ribbons of width $\Delta$.

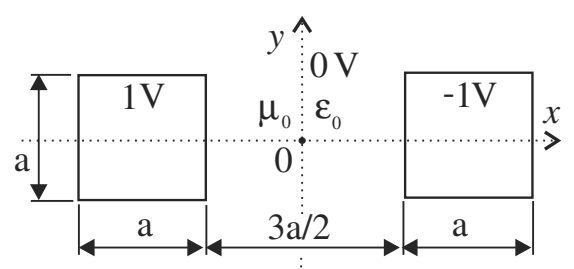

Fig. 1. Cross-section of the conductors

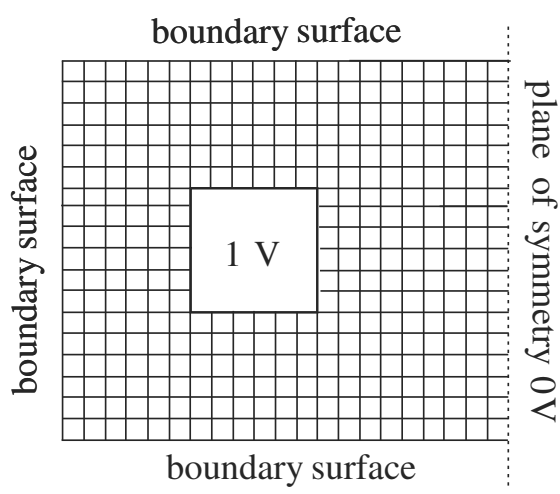

Fig. 2. The grid with square mesh which encloses only left conductor

The calculation of the potentials in the nodes on the boundary now becomes completely trivial, because the surface density of the charges is known on each one of ribbons and is 
given as [7]

$$
V=\sum_{i=1}^{2 n} \frac{1}{4 \pi \varepsilon_{0}} \int_{S_{i}} \frac{\sigma_{i} d s}{r^{2}}
$$

In eq. (6), $S_{i}$ is the surface of the $i$-th charged ribbon on the conductor surface. The total number of ribbons is $2 n, n$ on one and $n$ on the other conductor. Instead of direct calculation of the potentials in the nodes on the boundary by the means of the integral equation (6), and bearing in mind both the dimensions of the cell and the distance of the boundary surface from the conductor, the calculation of the potential can be simplified without significant error.



$$
\begin{gathered}
E_{1}=\frac{1}{2}\left[\left(-\frac{V_{C}-V_{A}}{\Delta}\right)+\left(-\frac{V_{D}-V_{B}}{\Delta}\right)\right] \\
E_{2}=\frac{1}{2}\left[\left(-\frac{V_{A}-V_{0}}{\Delta}\right)+\left(-\frac{V_{B}-V_{0}}{\Delta}\right)\right] \\
E_{3}=\frac{1}{2}\left(3 E_{2}-E_{1}\right) \Rightarrow \\
\sigma=\varepsilon_{0} E_{3} \Rightarrow Q^{\prime}=\sigma \Delta
\end{gathered}
$$

Fig. 3. The determination of the surface charge density on a conductor surface

DIRICHLET'S condition

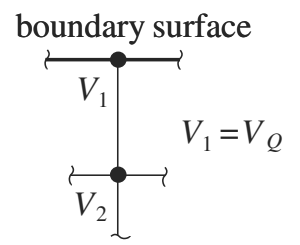

Fig. 4. With the Dirichlet's condition

Total charge of particular ribbons can be placed in the charged thread with linear charge of $Q^{\prime}=\sigma \Delta$, placed exactly in the center of a corresponding ribbon. Symmetrically, in relation to the equipotential plane with the potential $V=0$ (Fig. 1), the same thread exists on the other conductor, but the linear charge is of opposite sign, $-Q^{\prime}$. For above mentioned pair of threads, the potential is now calculated in relation to the reference point on the symmetric plane, in the point with coordinates $(x, y)$

$$
V_{i}(x, y)=\frac{Q_{i}^{\prime}}{2 \pi \varepsilon_{0}} \ln \sqrt{\frac{\left(y+y_{j}\right)^{2}+\left(x+x_{j}\right)^{2}}{\left(y-y_{j}\right)^{2}+\left(x-x_{j}\right)^{2}}}
$$

and the potential in every point in all $n$ pairs of threads is given by the expression

$$
V_{i}(x, y)=\sum_{i=1}^{n} V_{i}(x, y)
$$

where $x_{j}$ and $y_{j}$ i.e. $x_{j}$ and $-y_{j}$ are the coordinates of the points which define the position of the two infinite and straight threads with linear charge of $Q_{i}^{\prime}$ and $-Q_{i}^{\prime}$ which are normal to the $x-0-y$ plane [8]. For the same pair of threads, the components of the electrical field, in the direction of $x$ - and $y$-axis, in the points with the coordinates $(x, y)$, are

$$
E_{i x}(x, y)=\frac{Q_{i}^{\prime}}{2 \pi \varepsilon_{0}}\left[\frac{x-x_{j}}{\left(y-y_{j}\right)^{2}+\left(x-x_{j}\right)^{2}}-\frac{x+x_{j}}{\left(y-y_{j}\right)^{2}+\left(x+x_{j}\right)^{2}}\right]
$$




$$
E_{i y}(x, y)=\frac{Q_{i}^{\prime}}{2 \pi \varepsilon_{0}}\left[\frac{y-y_{j}}{\left(y-y_{j}\right)^{2}+\left(x-x_{j}\right)^{2}}-\frac{x+x_{j}}{\left(y-y_{j}\right)^{2}+\left(x+x_{j}\right)^{2}}\right],
$$

and the components of the electrical field which originate from the whole system of $n$ pairs of threads at the same point are

$$
\begin{aligned}
& E_{x}(x, y)=\sum_{i=1}^{n} E_{i x}(x, y), \\
& E_{y}(x, y)=\sum_{i=1}^{n} E_{i y}(x, y) .
\end{aligned}
$$

After one iteration, the value of the potential on the boundary is replaced by the value calculated in (8). The procedure of the alternate implementation of the iterative process, according to the expression (5) and the correction of boundary values of the potential (expression (8)), is continued until the sum of modules of the potential differences in all nodes in two successive iterations, $n^{\text {th }}$ and $(n+1)^{s t}$, becomes less than $10^{-5} \mathrm{~V}$ i.e.

$$
\sum_{i} \sum_{j}\left|V_{i j}^{n}-V_{i j}^{(n+1)}\right|<10^{-5} \mathrm{~V}
$$

Numerical calculations are repeated for different distances of the boundary surface from the conductor and the cells has always been square. For the sake of comparison, the values of the potential in selected nodes will be comparatively shown in the table, together with the potential values in the same nodes obtained by the implementation of different conditions for different grid dimensions.

\subsection{Limiting the mesh by the implementation of Dirichlet's and Neumann's boundary conditions applied on two close points}

The implementation of Neumann's and Dirichlet's conditions in two close points differs from the implementation of pure Dirichlet's condition only in the way of correction of the potential values after each iteration. The determination of the surface density of charges on the conductors is the same.

In Fig. 5 the detail from Fig. 2 is shown. The part of a boundary line is shown as well as three nodes, signed as $V_{1}, V_{2}$ and $V_{3}$. The normal component of the electric field on the boundary can be calculated by numerical differentiation as

$$
E_{n}=-\frac{V_{1}-V_{2}}{\Delta},
$$

or, directly based on the known distribution of charges on the conductor surfaces.

The distance between the boundary and the point in which the normal component of the field is calculated is the half of the cell length, i.e. $\frac{\Delta}{2}$.

The field component in the same point can be calculated based on the known distribution of charges on the conductor surfaces. As above, it is also approximated that the total charge 
on the ribbons, between every two pairs of nodes, is replaced by the charged thread placed in the center of ribbon.

NEUMANN'S + DIRICHLET'S conditions in two close points

$$
\begin{aligned}
& \stackrel{\text { boundary surface }}{\stackrel{V_{1}}{\sim} E_{\mathrm{n}}} \quad E_{n}=-\frac{V_{1}-V_{2}}{\Delta} \quad \text { NEUMANN } \\
& \stackrel{\longrightarrow}{V_{2}} \longrightarrow \quad E_{n}=E_{Q^{\prime}} \Rightarrow
\end{aligned}
$$



Fig. 5. With the Neumann's and Dirichlet's conditions in two close points

By equaling the normal components of the field on the boundary, obtained by the numerical differentiation of $E_{n}$ from the eq. (14) (shown as $E_{Q^{\prime}}$ ), the new potential value in the node on the boundary is obtained ( 5)

$$
V_{1}=V_{2}-\Delta E_{Q^{\prime}}
$$

The correction of the boundary values is performed in described way in all boundary nodes after each iteration. The criterion for ending the iterative process is the same as in the implementation of the Dirichlet's condition and is given by eq. (13).

\subsection{Limiting the mesh by the implementation of Cauchy's boundary condition}

Cauchy's boundary condition is very rarely used in the solution of electrostatic problems. It really means that the fulfilling of both the Dirichlet's and Neumann's conditions is needed in the same point.

Basically, everything said for the two cases also holds now. The calculation of the charges distributed on the conductor surfaces is the same. The same approximation is also used - the charged ribbon is replaced by the charged thread. The main difference is only in connecting the normal component of the field on the boundary in the node $V_{1}$ (Fig. 6) with the potential in the neighbouring two nodes on the boundary. By numerical extrapolation, the following link between the potential in three close nodes and the normal components of the field on the boundary is obtained

$$
V_{1}=\frac{1}{3}\left(4 V_{2}-V_{3}-2 \Delta E_{n}\right)
$$

On the other hand, the normal component of the field can be calculated as in 4.1. Based on the known distribution of the charges from eqs. (11) and (12), let us sign the directly calculated normal component of the field as $E_{Q^{\prime}}$. Taking into account that $E_{n}=E_{Q^{\prime}}$, the equation (16) becomes 


\section{CAUCHY'S condition}

$$
\begin{aligned}
& \begin{array}{l}
\text { boundary } \\
\text { surface }
\end{array} \\
& V_{1}=\frac{1}{3}\left(4 V_{2}-V_{3}-2 \Delta E_{n}\right) \\
& E_{n}=E_{Q^{\prime}} \Rightarrow \\
& V_{1}=\frac{1}{3}\left(4 V_{2}-V_{3}-2 \Delta E_{Q^{\prime}}\right)
\end{aligned}
$$

Fig. 6. With the Cauchy's condition

$$
V_{1}=\frac{1}{3}\left(4 V_{2}-V_{3}-2 \Delta E_{Q^{\prime}}\right)
$$

According to the condition (17), the corrections of the potential are performed after each iteration. The end of the iterative process is again realized by the same criterion, eq. (13).

\section{Numerical results}

The numerical example has been solved in several variants. The position of the wire conductor and the dimensions from Fig. 1 have not been changed. Only the grid dimensions were changed. In all variants of the solution, with many grids, the potentials in the points defined in Fig. 7 have been calculated, in order to easily compare of the results. In all variants the finite difference method of the first kind is used with mesh dimensions of $\Delta \times \Delta$.

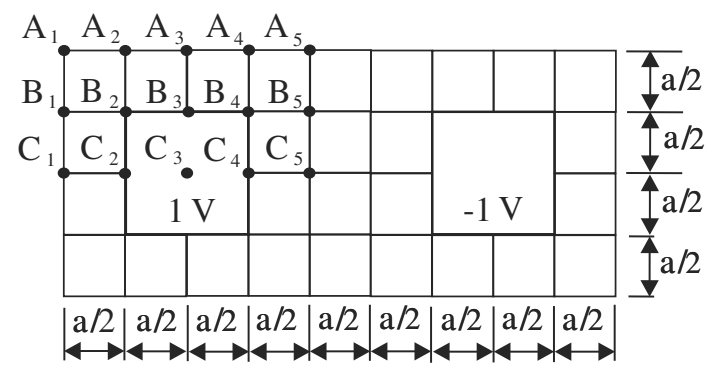

Fig. 7. Defining the position of the points in which the potential values are presented in tables

The first set of the potential values, given in Tab. 1, is related to the smallest grid - with the least number of nodes. That grid, defined in Fig. 8, has cells $\Delta \times \Delta$ where $\Delta=\frac{a}{8}$. In the table, the potential values in the points $A_{1}-A_{5}, B_{1}-B_{5}$ and $C_{1}-C_{5}$ are presented in Fig. 7. The results for Dirichlet's, Neumann's and Cauchy's boundary conditions are separately presented. 




Fig. 8. Defining the grid size by using the parameters $m$ and $k$ (see Figs. 1 and 2)

Table 1. Grid parameters: $k=5, m=8$ and $\Delta=\frac{a}{8}$ (Fig. 8)

\section{DIRICHLET}

\begin{tabular}{|c|c|c|c|c|c|}
\hline \multirow{2}{*}{$\begin{array}{c}\text { point } \\
\downarrow\end{array}$} & \multicolumn{5}{|c|}{ index } \\
\cline { 2 - 6 } & 1 & 2 & 3 & 4 & 5 \\
\hline$A$ & 0.5825 & 0.6539 & 0.6397 & 0.4866 & 0.1760 \\
\hline$B$ & 0.7020 & 1 & 1 & 1 & 0.2774 \\
\hline$C$ & 0.7480 & 1 & 1 & 1 & 0.3156 \\
\hline
\end{tabular}

NEUMANN+DIRICHLET

\begin{tabular}{|c|c|c|c|c|c|}
\hline point & \multicolumn{5}{|c|}{ index } \\
\cline { 2 - 6 }$\downarrow$ & 1 & 2 & 3 & 4 & 5 \\
\hline$A$ & 0.6794 & 0.7330 & 0.7059 & 0.5434 & 0.1987 \\
\hline$B$ & 0.7771 & 1 & 1 & 1 & 0.2837 \\
\hline$C$ & 0.8118 & 1 & 1 & 1 & 0.3175 \\
\hline
\end{tabular}

\section{CAUCHY}

\begin{tabular}{|c|c|c|c|c|c|}
\hline \multirow{2}{*}{$\begin{array}{c}\text { point } \\
\downarrow\end{array}$} & \multicolumn{5}{|c|}{ index } \\
\cline { 2 - 6 } & 1 & 2 & 3 & 4 & 5 \\
\hline$A$ & 0.6791 & 0.7314 & 0.7044 & 0.5397 & 0.1977 \\
\hline$B$ & 0.7761 & 1 & 1 & 1 & 0.2833 \\
\hline$C$ & 0.8115 & 1 & 1 & 1 & 0.3174 \\
\hline
\end{tabular}

The second set of the potential values, given in Tab. 1, is related to the grid with dimensions defined in Fig. 8. The cells are $\Delta \times \Delta$ where $\Delta=\frac{a}{16}$. 
Table 2. Grid parameters: $k=10, m=16$ and $\Delta=\frac{a}{16}$ (Fig. 8)

\section{DIRICHLET}

\begin{tabular}{|c|c|c|c|c|c|}
\hline point & \multicolumn{5}{|c|}{ index } \\
\cline { 2 - 6 }$\downarrow$ & 1 & 2 & 3 & 4 & 5 \\
\hline$A$ & 0.5899 & 0.6601 & 0.6463 & 0.4908 & 0.1780 \\
\hline$B$ & 0.7091 & 1 & 1 & 1 & 0.2756 \\
\hline$C$ & 0.7546 & 1 & 1 & 1 & 0.3149 \\
\hline
\end{tabular}

NEUMANN+DIRICHLET

\begin{tabular}{|c|c|c|c|c|c|}
\hline point & \multicolumn{5}{|c|}{ index } \\
\cline { 2 - 6 }$\downarrow$ & 1 & 2 & 3 & 4 & 5 \\
\hline$A$ & 0.6509 & 0.7098 & 0.6878 & 0.5248 & 0.1916 \\
\hline$B$ & 0.7559 & 1 & 1 & 1 & 0.2794 \\
\hline$C$ & 0.7953 & 1 & 1 & 1 & 0.3116 \\
\hline
\end{tabular}

CAUCHY

\begin{tabular}{|c|c|c|c|c|c|}
\hline point & \multicolumn{5}{|c|}{ index } \\
\cline { 2 - 6 }$\downarrow$ & 1 & 2 & 3 & 4 & 5 \\
\hline$A$ & 0.6520 & 0.7104 & 0.6886 & 0.5253 & 0.1919 \\
\hline$B$ & 0.7565 & 1 & 1 & 1 & 0.2795 \\
\hline$C$ & 0.7959 & 1 & 1 & 1 & 0.3160 \\
\hline
\end{tabular}

The third set of the potential values, given in Tab. 3, is related to the grid with dimensions defined in Fig. 8. The cells are $\Delta \times \Delta$ where $\Delta=\frac{a}{16}$.

Table 3. Grid parameters: $k=16, m=16$ and $\Delta=\frac{a}{16}$ (Fig. 8)

\section{DIRICHLET}

\begin{tabular}{|c|c|c|c|c|c|}
\hline \multirow{2}{*}{$\begin{array}{c}\text { point } \\
\downarrow\end{array}$} & \multicolumn{5}{|c|}{ index } \\
\cline { 2 - 6 } & 1 & 2 & 3 & 4 & 5 \\
\hline$A$ & 0.5914 & 0.6647 & 0.6532 & 0.4989 & 0.1816 \\
\hline$B$ & 0.7122 & 1 & 1 & 1 & 0.2765 \\
\hline$C$ & 0.7579 & 1 & 1 & 1 & 0.3152 \\
\hline
\end{tabular}


NEUMANN+DIRICHLET

\begin{tabular}{|c|c|c|c|c|c|}
\hline \multirow{2}{*}{$\begin{array}{c}\text { point } \\
\downarrow\end{array}$} & \multicolumn{5}{|c|}{ index } \\
\cline { 2 - 6 } & 1 & 2 & 3 & 4 & 5 \\
\hline$A$ & 0.6364 & 0.6969 & 0.6764 & 0.5145 & 0.1874 \\
\hline$B$ & 0.7469 & 1 & 1 & 1 & 0.2782 \\
\hline$C$ & 0.7884 & 1 & 1 & 1 & 0.3157 \\
\hline
\end{tabular}

CAUCHY

\begin{tabular}{|c|c|c|c|c|c|}
\hline point & \multicolumn{5}{|c|}{ index } \\
\cline { 2 - 6 }$\downarrow$ & 1 & 2 & 3 & 4 & 5 \\
\hline$A$ & 0.6337 & 0.6975 & 0.6769 & 0.5148 & 0.1875 \\
\hline$B$ & 0.7469 & 1 & 1 & 1 & 0.2783 \\
\hline$C$ & 0.7884 & 1 & 1 & 1 & 0.3157 \\
\hline
\end{tabular}

\section{Conclusion}

One of the most significant deficiencies of the finite difference method in solving the problems of the field calculation (or similar) in open space is that it's calculation domain needs to be artificially limited. This introduces an additional error in the calculation whose value was difficult to estimate. In this work it was demonstrated, both theoretically and on the example, how one of the main deficiencies of the finite difference methods can be overcome in a numerically exact way and in that way significantly improve the range of implementation of the method as well as the quantity of the calculation.

The analyzed numerical example has shown that. by the implementation of the classical boundary conditions (Dirichlet's, Neumann's, etc.). the area of the numerical calculation can be exactly limited for solving problems in open space. The results of the calculation are in precisely agreement with the results from reference [10].

The results obtained by the implementation of Cauchy's, Dirichlet's and Neumann's conditions in two close points are in better agreement than the results obtained by the implementation of only one boundary condition - which was expected.

\section{References}

[1] S. J. FARLOW, Partial differential equations for scientists and engineers, New York, J. Wiley and Sons, 1982.

[2] R. H. Gordon, S. H. Fook, A finite difference approach that employs an asymptotics boundary condition on a rectangular outer boundary for modeling two-dimensional transmissonal line structures, IEEE Trans Microwave Theory Tech., Vol.41, No 8, Aug 1993. pp.1280-1286.

[3] Z. HAZNADAR, M. LOVRENJAK, The field calculation by using the finite difference method (in croatian), Zagreb, Elektrotehnika, No 5, 1971. pp. 33-47. 
[4] J. JIN, The finite element method in electromagnetics, New York, J.Wiley and Sons, 1993.

[5] H. OKUbo, M. IKedA, M. HondA, T. YANARI, Electric field analysis by combination method, IEEE trans., Vol. PAS-101, No 10, Oct.1982. pp.4039-4048.

[6] H. Okubo, M. Ikeda, M. Honda, S. Menju, Combination method for electric field calculation, Third Inter. Symp. on High Voltage Engineering, Milan, 28-31. Aug. 1979. paper 23.14 .

[7] B. D. Popović, Electromagnetics (in serbian), Beograd, Građevinska knjiga, 1980.

[8] H. Singer, H. Steinbigler, P. Weiss, A charge simulation method for the calculation of high voltage fields, IEEE Trans. Pow. App, Sys, PAS -95. pp. 1660-1668. 1974.

[9] H. STEInBIGLER, Combined application of finite element method and charge simulation method for the computation of electric fields, Third Int. Symp. On High voltage Engineering. Milan. 28-31. Aug. 1979. paper 11.11.

[10] C. G. Williams, G. K. CAMBRELl, Efficient numerical solution of unbounded fields problems, Electronics letters, Vol.8. No 7, 4th May 1972. pp. 247-248.

[11] O.C. ZiEnkiewicz, K. Morgan, Finite elements and approximation, New York, J. Wiley and Sons, 1983. 